In Situ

Revue des patrimoines

\section{In Situ}

Revue des patrimoines

$28 \mid 2016$

Le moulage. Pratiques historiques et regards contemporains

\title{
Du plâtre et de la poésie. Les moulages d'après Michel-Ange à l'École des beaux-arts de Paris
}

Of Plaster and Poetry. The plaster casts of Michelangelo's sculptures at the

Paris École des Beaux-Arts

\section{Emmanuel Schwartz}

\section{OpenEdition}

\section{Journals}

Édition électronique

URL : http://journals.openedition.org/insitu/12411

DOI : $10.4000 /$ insitu. 12411

ISSN : 1630-7305

\section{Éditeur}

Ministère de la Culture

\section{Référence électronique}

Emmanuel Schwartz, «Du plâtre et de la poésie. Les moulages d'après Michel-Ange à l'École des beauxarts de Paris », In Situ [En ligne], 28 | 2016, mis en ligne le 16 mars 2016, consulté le 21 décembre 2020. URL : http://journals.openedition.org/insitu/12411; DOI : https://doi.org/10.4000/insitu.12411

Ce document a été généré automatiquement le 21 décembre 2020.

\section{(i) $\odot$

In Situ Revues des patrimoines est mis à disposition selon les termes de la licence Creative Commons Attribution - Pas d'Utilisation Commerciale - Pas de Modification 4.0 International. 


\section{Du plâtre et de la poésie. Les moulages d'après Michel-Ange à l'École des beaux-arts de Paris}

Of Plaster and Poetry. The plaster casts of Michelangelo's sculptures at the Paris École des Beaux-Arts

\section{Emmanuel Schwartz}

Communication présentée lors des journées d'étude « Le moulage. Pratiques historiques et regards contemporains » organisées par la Cité de l'architecture et du patrimoine et le musée du quai Branly, les 14 et 15 novembre 2012.

(fig. 1) 


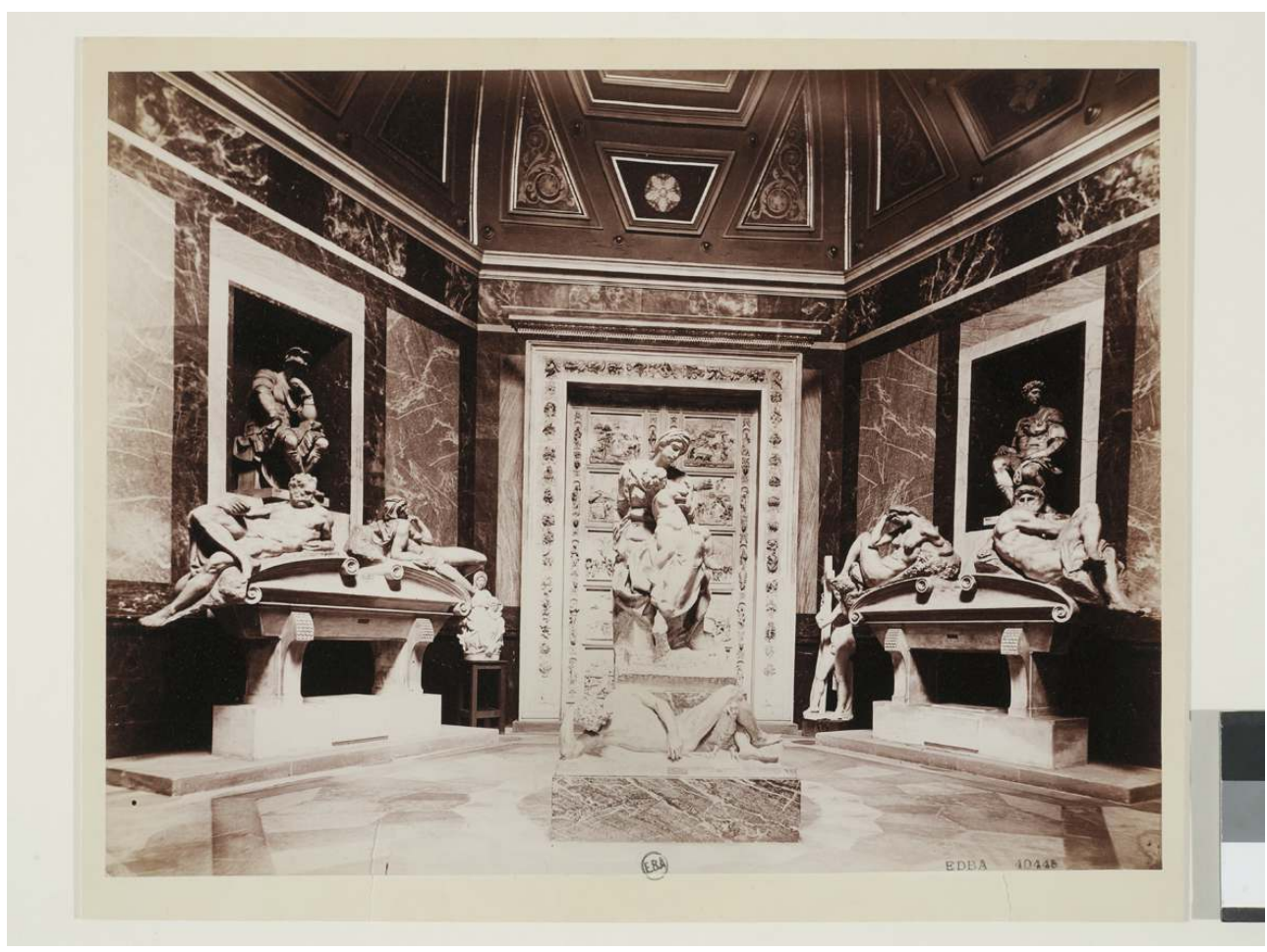

Anonyme. La chapelle de Michel-Ange dans l'École des beaux-arts de Paris. Au milieu : L'Adonis mourant d'après Vincenzo di Raffaello de' Rossi. Photographie, 1919. Paris, École nationale supérieure des beaux-arts, $\mathrm{PH} 149$.

Phot. Lapelerie, Jean-Michel. (c) École nationale supérieure des beaux-arts.

2 Les moulages acquièrent parfois une signification que les originaux dont ils sont tirés ignorent. Pendant un siècle, en France, quelques moulages d'après Michel-Ange offrirent des modèles artistiques, moraux, littéraires et philosophiques. Il fallut pour cela, outre une présentation spectaculaire, une longue préparation historique et quelques coïncidences.

\section{Points d'histoire}

3 Michel-Ange eut de son vivant en France des admirateurs royaux. Ses œuvres arrivèrent nombreuses, originaux et copies ; beaucoup se sont perdues. François $\mathrm{I}^{\mathrm{er}}$ commanda des moulages du Cristo della Minerva et de la Pietà du Vatican. Un an avant de mourir, il écrivait à Michel-Ange vouloir en « orner une de [ses] chapelles comme de choses que l'on m'a assuré estre des plus exquises et excellentes en votre $\operatorname{art}^{1}$. » L'esprit de Michel-Ange perce chez quelques artistes de la Renaissance française; le gisant de Henri II par Germain Pilon dérive du Christ de la Pietà. Un destin capricieux décida qu'une chapelle parisienne abriterait successivement le tombeau de François $\mathrm{I}^{\mathrm{er}}$, venu de Saint-Denis, le gisant de son fils Henri, puis un temple à Michel-Ange dominé par la Piétà - du musée de la Révolution à l'École de la République, soit l'ancienne chapelle de la reine Margot de 1610 convertie en mausolée de François $\mathrm{I}^{\mathrm{er}}$ puis en tempietto de Michel-Ange.

4 Lorsque l'Académie royale fut fondée, une collection de plâtres y fut accumulée. MichelAnge n'y était pas le bienvenu. Fréart de Chambray vouait au nom de l'ordre moral une 
haine irréconciliable à ce « fanfaron, impie, faux chrétien, libertin : de Raphaël et MichelAnge, on pourrait dire en vérité que l'un est le Bon, et l'autre le Mauvais Ange ${ }^{2}$ [de la Peinture] ». Un seul talent était reconnu à Michel-Ange, l'anatomie, mais avec « une certaine dureté affectée dans sa manière de desseigner ». «Il affectoit de charger trop les muscles de ses figures, et d'en outrer les attitudes ${ }^{3}$.» On lui attribua des statuettes d'écorchés aux muscles saillants et aux extravagantes contorsions ${ }^{4}$, premiers modèles dits de Michel-Ange proposés aux élèves (fig. 2).

\section{Figure 2}

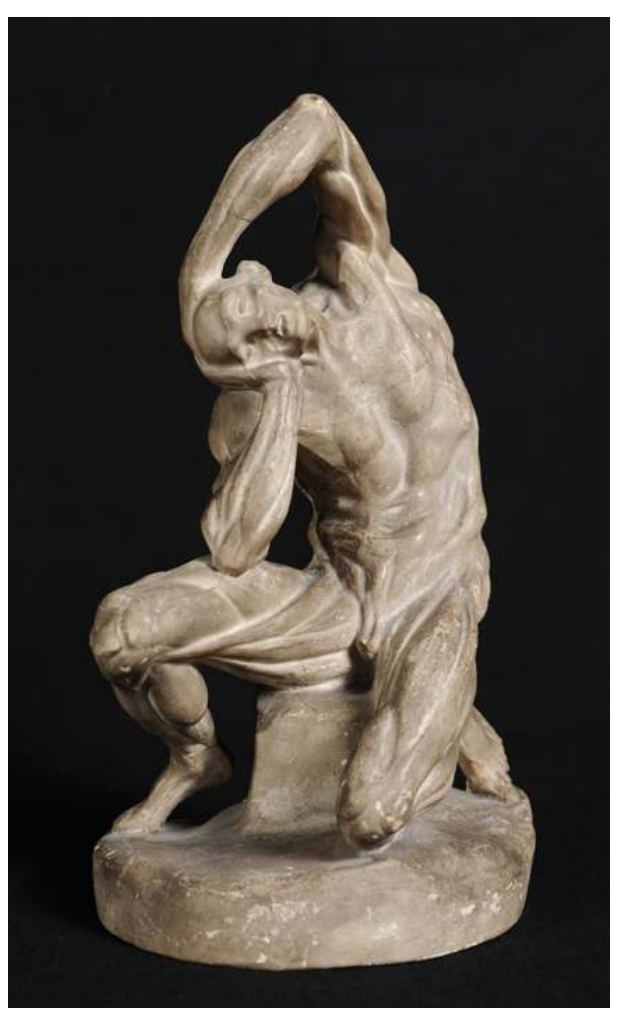

Anonyme. Écorché, dit de Michel-Ange, XVIe siècle, moulage en plâtre. Paris, École nationale supérieure des beaux-arts, MU 11992.

Phot. Lapelerie, Jean-Michel. (c) École nationale supérieure des beaux-arts.

5 Les révolutions donnèrent à Michel-Ange une seconde chance française; elles convergèrent vers le même et unique lieu, où se trouve maintenant l'École des beaux-arts de Paris, héritière de l'Académie royale, et vers un seul genre, jugé mineur partout, sauf dans les colloques, le moulage. La première révolution, dans la grande tourmente, fut l'œuvre des esclaves Strozzi. Ils étaient restés dans la collection privée des Richelieu. Un individu seul, Alexandre Lenoir, les sauva de la destruction en 1793. Le Captif rebelle, le Captif mourant : c'était la prise de la Bastille en deux titres (fig. $\mathbf{3}$ a et b). 
Figure 3ab

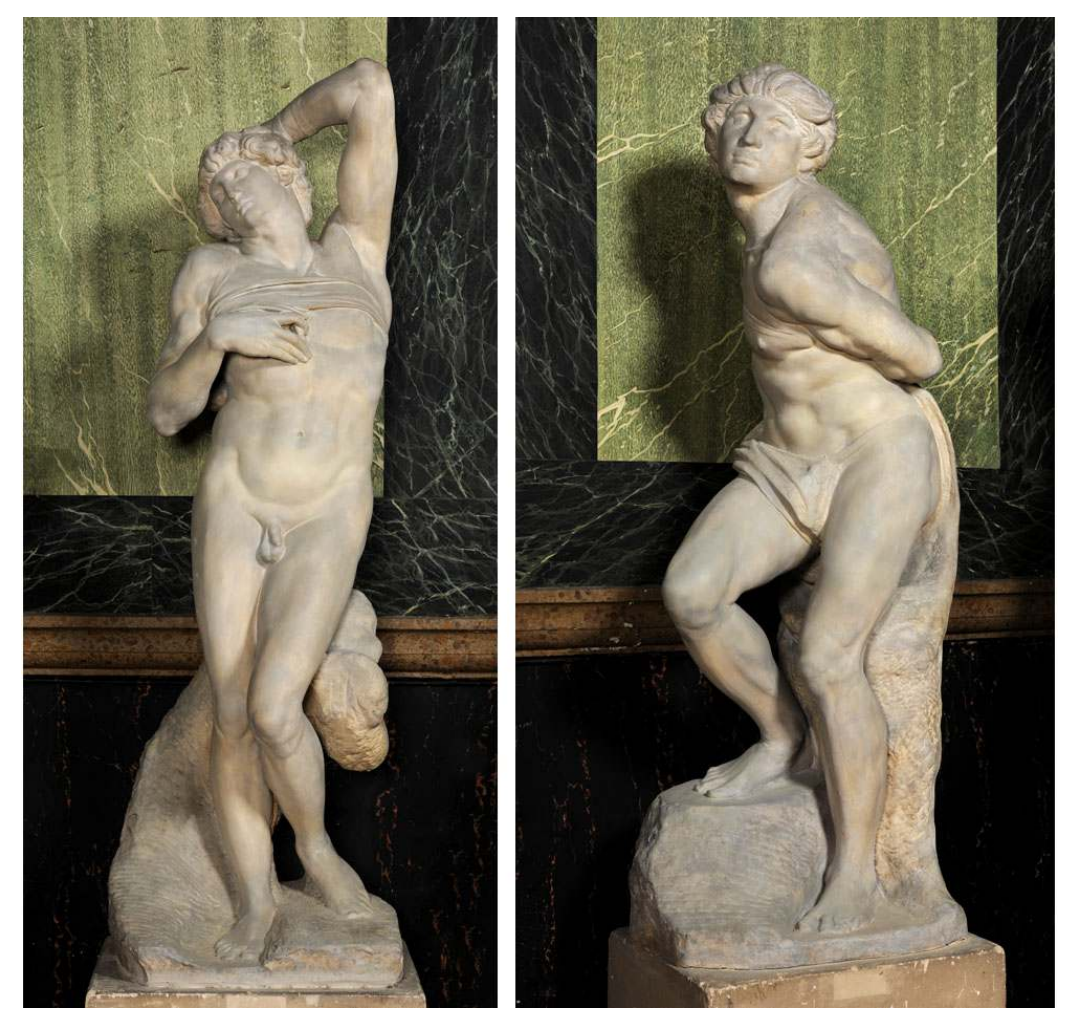

Antonio Banchelli. Les Esclaves de Michel-Ange, 1838, moulage. Paris, École nationale supérieure des beaux-arts, MU 3663(ab).

Phot. Lapelerie, Jean-Michel. (C École nationale supérieure des beaux-arts.

Dans le faubourg Saint-Germain, dans l'église déchristianisée des Petits-Augustins, dans son musée des Monuments français, le premier, l'authentique, le modèle de tous les autres, musée des rois déchus, des dieux morts et des artistes libres, Lenoir recueillit ces «deux morceaux, uniques en France, [qui...] portent avec eux l'idée du sublime et du terrible $»^{5}$. Le Muséum du Louvre les exigea. Lenoir fit une place d'honneur à MichelAnge, avec Winckelmann seul étranger accepté dans son musée, cuit dans la terre par Guillaume Boichot (fig. 4), adaptation du buste de Daniele da Volterra. L'odeur sulfureuse du musée sacrilège et jacobin, fermé par les Bourbons en 1817, persista dans l'École des beaux-arts qui lui succéda aux Petits-Augustins ; elles y sont encore - l'École et l'odeur de soufre. Les Esclaves furent les premiers moulages modernes produits par l'atelier du Louvre. 
Figure 4

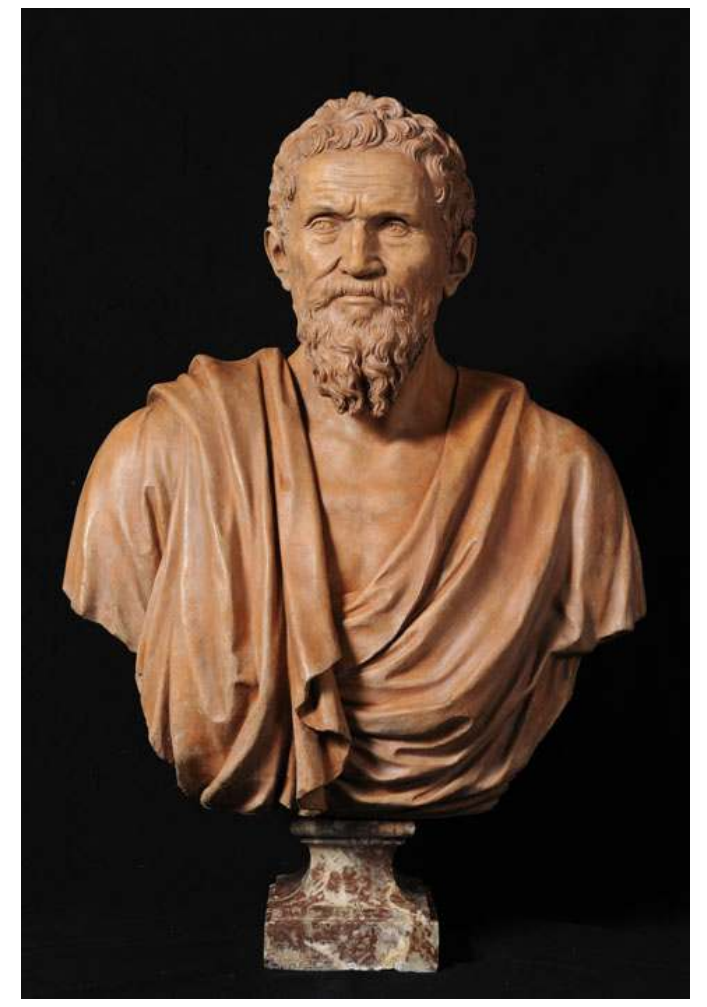

Guillaume Boichot (1735-1814). Buste de Michel-Ange, c. 1800, terre cuite. Paris, École nationale supérieure des beaux-arts, MU 1256.

Phot. Lapelerie, Jean-Michel. ( C École nationale supérieure des beaux-arts.

7 La révolution esthétique, exaltée par Edmond Burke, déclara sublime en France la mélancolie et la terribilità, donc Michel-Ange. Une troisième révolution mit les vaincus et les victimes au-dessus des vainqueurs et des survivants, au moins en art: le mythe de l'artiste maudit naissait sur le corps supplicié d'André Chénier. Le sculpteur solitaire de Florence fut promu héros d'une génération sans dieu ni maître. Or, précisément, les enfants du siècle, Stendhal, Delacroix, Michelet, Dumas, fréquentaient, parmi d'autres mauvais lieux, l'École des beaux-arts. Leur voix y résonne encore.

\section{Création d'une collection scolaire}

Chose surprenante : il revint à un ministre réactionnaire et brutal, l'exact opposé des révolutionnaires, l'antithèse de Michel-Ange, d'édifier un temple au Divino. Le Divino, voilà un surnom bien différent de ceux de M. Thiers, dit Crapaud Venimeux, dit César en raccourci, dit Père Transnonain, dit Polichinelle tricolore, dit Taupe à lunettes, dit enfin Adolphe le petit. Le petit Adolphe de la France se livra à la provocation suprême, en pleine école académique; il fit assembler par une de ses créatures, le conservateur de l'École, Hippolyte Peisse, une collection de moulages d'après Michel-Ange. Thiers et Peisse voulaient que la copie récente du Jugement dernier (fig. 5) fût confrontée «avec presque toute son œuvre en sculpture. 
Figure 5

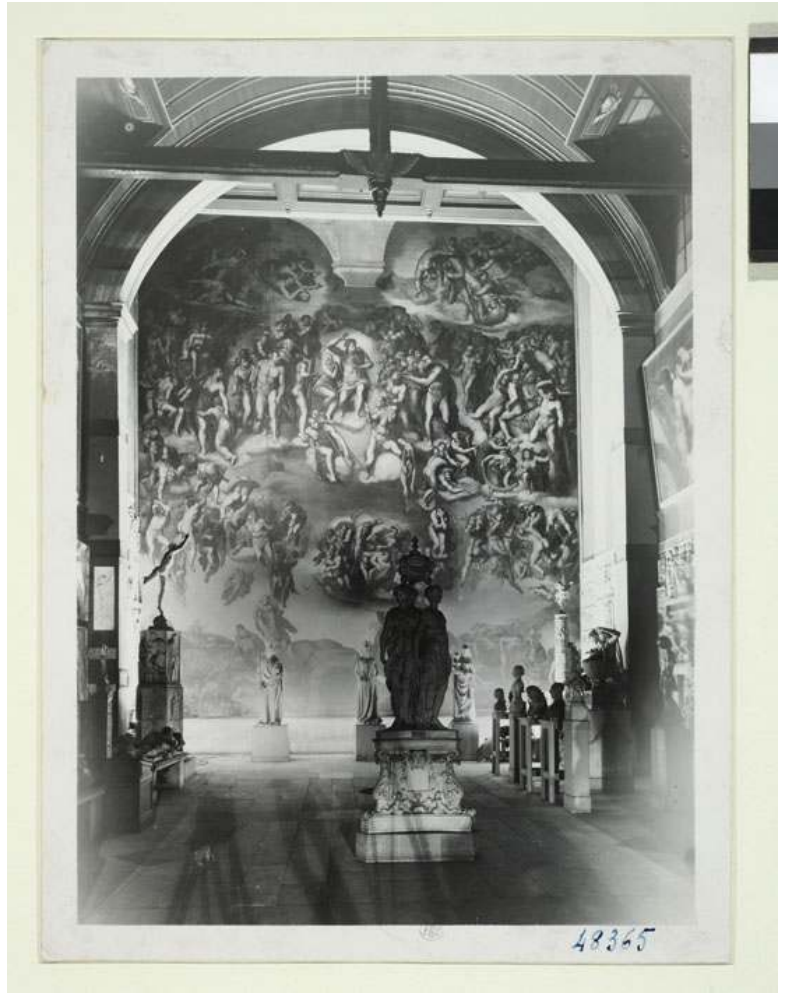

L. Borremans. L'église des Petits-Augustins avec la copie du Jugement dernier, 1936, photographie. Paris, École nationale supérieure des beaux-arts, $\mathrm{PH} 8451$.

Phot. Lapelerie, Jean-Michel. (c) École nationale supérieure des beaux-arts.

9 Ainsi, ce que l'art moderne offre de plus grand, Michel-Ange, sera là tout entier. » Peisse anticipait sur le présent ouvrage et colloque : «La collection des plâtres est la seule [...] qui systématiquement formée et classée, soit propre à influer utilement sur l'art et sur le goût public ${ }^{6}$. " La chapelle de Margot fut donc vouée à l'artiste anathémisé par les bienpensants, tandis que l'architecte de l'École évoquait la Sixtine par la balustrade, à l'entrée de l'église exécrée. Le moulage du buste pour le tombeau de Michel-Ange veillait sur son sanctuaire (fig. 6) : Stendhal venait se recueillir devant « l'homme mélancolique et simple de l'église des Petits-Augustins ${ }^{7}{ }$. 


\section{Figure 6}

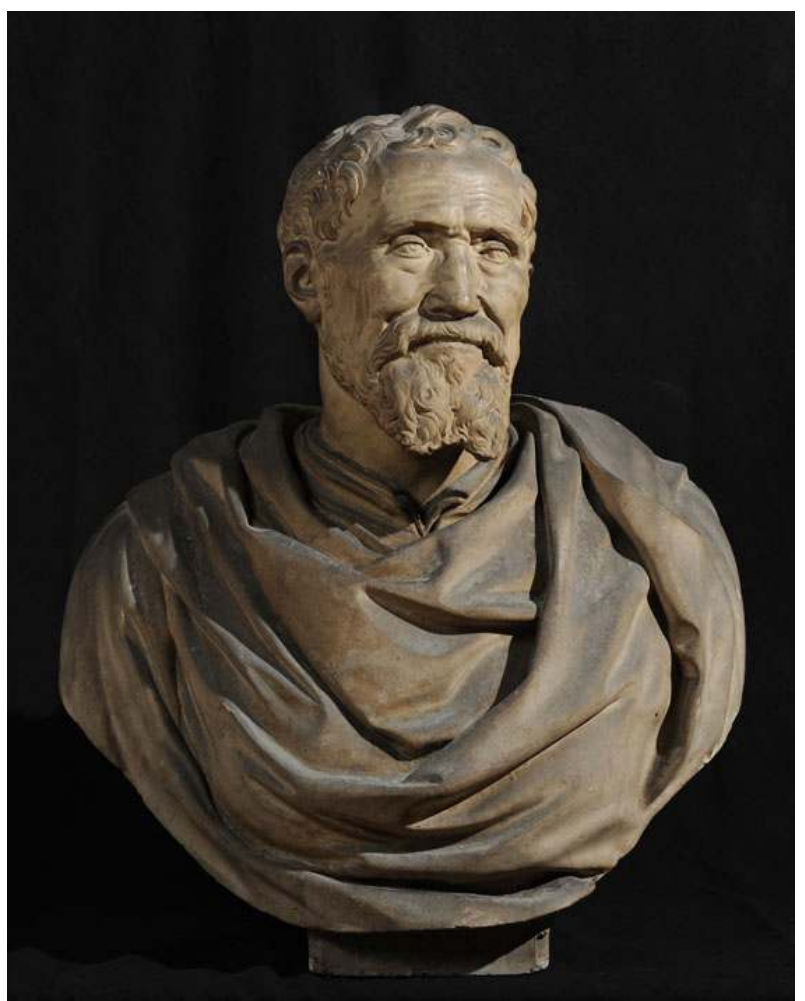

Antonio Banchelli et Clemente Papi. Buste de Michel-Ange, 1837, moulage d'après Battista di Domenico. Paris, École nationale supérieure des beaux-arts, MU 3668.

Phot. Lapelerie, Jean-Michel. (c) École nationale supérieure des beaux-arts.

Peisse partit acheter en Italie "les six sculptures des Médicis par Michel-Ange; son Moyse, son Bacchus, sa Pietà et d'autres moins importants; les fameuses portes de Florence par Lorenzo Ghiberti ${ }^{8}[. .]$.$» que Michel-Ange avait baptisées Portes d u$ Paradis. Les tombeaux des Médicis et de Jules II furent reconstitués ; le Moïse (fig. 7) attendit trente ans les Esclaves. 
Figure 7

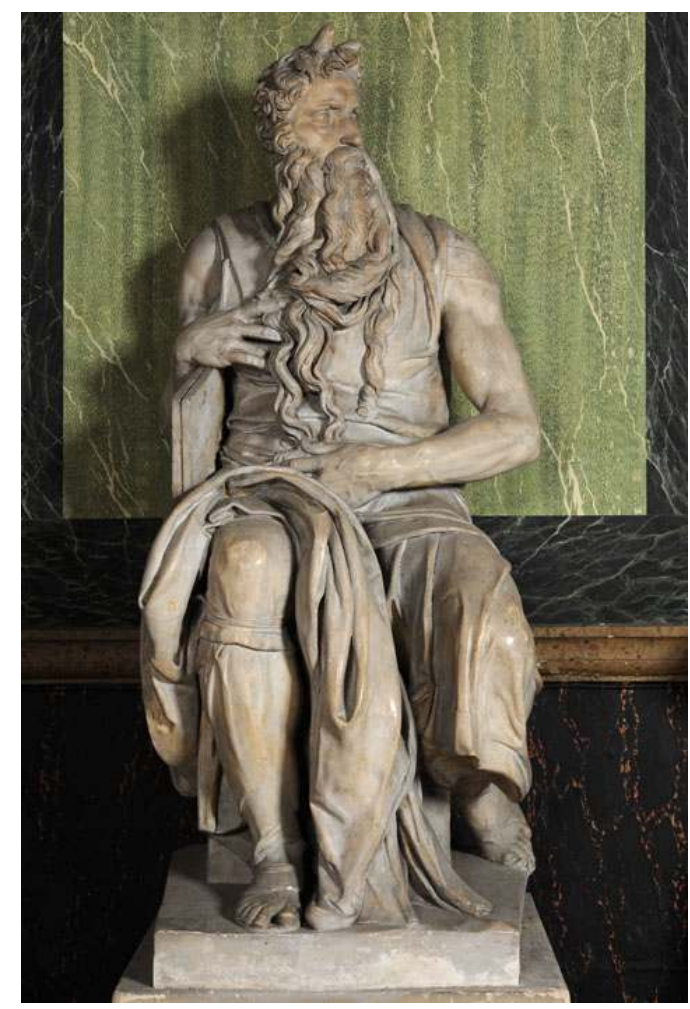

Le Moïse de Michel-Ange, 1836, moulage. Paris, École nationale supérieure des beaux-arts, MU 3664.

Phot. Lapelerie, Jean-Michel. (c) École nationale supérieure des beaux-arts.

La scénographie recréait celle de la Révolution, les gisants français de Saint-Denis, avec l'Henri II de Pilon, revenant - mais en plâtre. On était dans une école, une école d'art et d'artistes. Les professeurs, naturellement, ne faisaient rien, ne pensaient guère plus. Les jeunes Romantiques furent-ils attirés par le souvenir de la reine Margot, chère à leur cœur, qui avait élevé cette chapelle pour y sanctifier ses amours scandaleuses? Delacroix, sous sa voûte, incita les rudes tempéraments à affronter la leçon du génie ${ }^{9}$. Un Anglais, étudiant en art excentrique, William M. Thackeray, le futur auteur de La Foire aux Vanités, le plus brillant roman du siècle, envoya en 1839 , parmi ses croquis parisiens, ses réflexions «Sur [...] l'École française, accompagné d'anecdotes, d'illustrations et de considérations philosophiques", extrait d'une lettre à M. MacGilp, de Londres :

In the same school of the Beaux-Arts, where are to be found such a number of pale imitations of the antique, Monsieur Thiers (and he ought to be thanked for it) has caused to be placed a full-sized copy of The Last Judgment of Michael Angelo and a number of casts from statues by the same splendid hand. There is the sublime, if you please - a new sublime - an original sublime - quite as sublime as the Greek sublime. See yonder, in the midst of his angels, the Judge of the world descending in glory; and near him, beautiful and gentle, and yet indescribably august and pure, the Virgin by his side. There is the Moses, the grandest figure that ever was carved in stone. It has about it something frightfully Majestic, if one may so speak. In examining this, and the astonishing picture of the Judgement, or even a single figure of it, the spectator's sense amounts almost to pain. I would not like to be left in a room alone with the Moses. How did the artist live amongst them, and create them? How did he suffer the painful labour of invention? One fancies that he would have been scorched up, like Semele, by sights too tremendous for his vision to bear. One cannot imagine him, with our small physical endowments and weaknesses, a man 
like ourselves. As for the École royale des beaux-arts, then, and all the good its students have done, as students, it is stark naught ${ }^{10}$.

Le romancier artiste regardait le plâtre, il raisonnait en marbre, la plupart des élèves ne voyaient ni le marbre, ni le plâtre. Tout est là.

Quelques élèves pourtant vénéraient ces moulages, à l'égal d'objets de piété (fig. 8). Dans le sanctuaire profané, Jean-Baptiste Carpeaux obligea sa fiancée à s'agenouiller devant la Madone Médicis en plâtre. Il rapporta ce sacrilège en des termes dégoulinants de bigoterie : « Notre prière à sa belle Vierge m'a donné l'assurance que tous nos vœux seront accomplis. " Pour premier vœu à la mère de notre Sauveur, Carpeaux attendait un miracle : que le Divino transmît une parcelle de "son noble et sublime génie » à une demoiselle Amélie de Montfort, fille d'un marquis, général de brigade, qui plus est ${ }^{11}$. Carpeaux conçut à Rome son Ugolin en mêlant le Torse du Belvédère, que l'on appelait l'École de Michel-Ange et Laurent de Médicis le Pensieroso. Des copies de ces deux modèles existaient, l'antique en marbre et en plâtre, le moderne en moulage, dans l'École des beaux-arts. L'ancien élève mit un point d'honneur à présenter un modèle d'Ugolin en plâtre dans son École ; les professeurs se firent un devoir de l'éreinter.

Figure 8

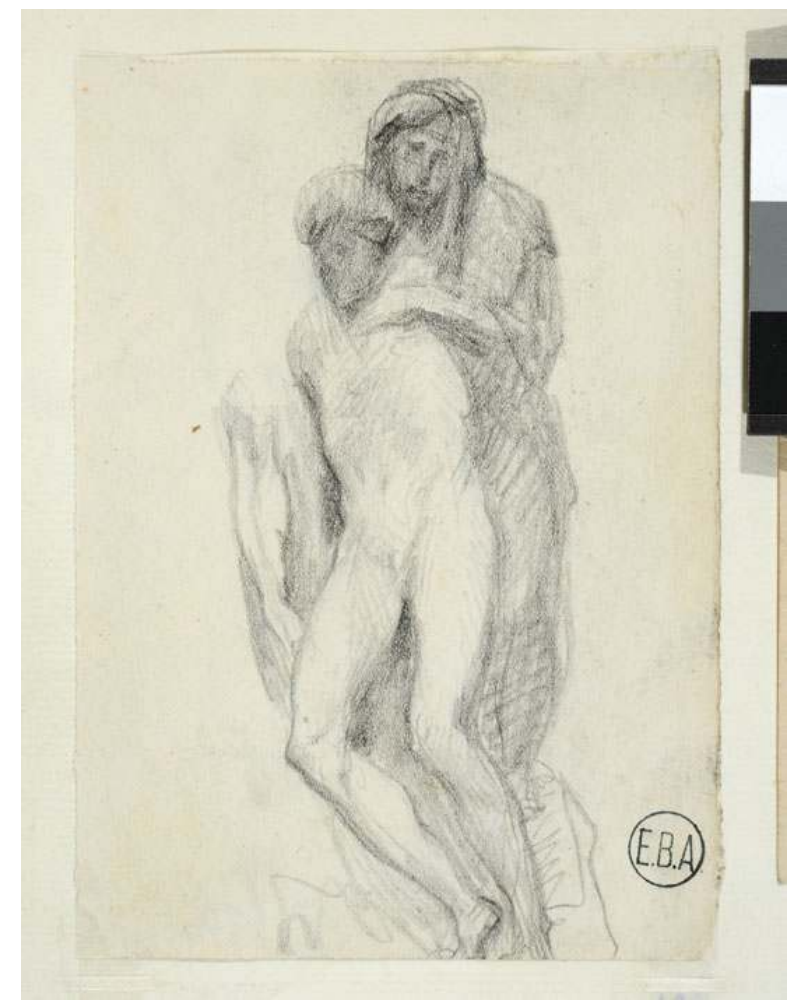

Jean-Baptiste Carpeaux. La Pietà Rondanini de Michel-Ange, c. 1869, dessin. Paris, École nationale supérieure des beaux-arts, EBA 1787-420.

Phot. Lapelerie, Jean-Michel. ( École nationale supérieure des beaux-arts.

\section{Questions d'histoire de l'art}

Les pèlerins de Michel-Ange abordaient les mêmes questions qu'avant la grande révolution, mais les réponses en étaient inversées. Le paganisme valait titre de noblesse 
morale. Théophile Gautier, subtil et définitif, tranchait : «Michel-Ange passe en général pour [...] violent et féroce de style, sauvage dans son faire, austèrement catholique et tout à fait semblable à Dante, sa grande admiration. Cependant, rien n'est plus faux. L'illustre rival de Raphaël est un artiste païen $»^{12}$. Jules Michelet, la voix de l'Histoire, l'Esprit de la Révolution, le verbe de Danton, mit les moulages de Michel-Ange très haut au-dessus des hommes et de Dieu :

Tout le monde a pu voir à Florence (ou à Paris, École des beaux-arts) les sublimes figures du Jour et de la Nuit, du Crépuscule et de l'Aurore, ce monument qui devint, sous la main du grand citoyen, le tombeau de la patrie même. La Nuit roule en son rêve une mer de honte et de misère. Mais l'Aurore! C'est bien pis: on sent qu'elle maudit son réveil et qu'elle a à la bouche un dégoût si amer, un fiel si déplaisant, qu'elle voudrait n'être jamais née. Ce qui fut plus tragique que le tragique monument, c'est que, quand il fut découvert, il n'eut personne pour le comprendre. Plus de Florence, plus de peuple, plus d'Italie. L'Académie est née. Un poète académique (nouveau fléau de ce pays) lance un madrigal à la Nuit :

'Dans sa douce attitude, elle dort ; ne la réveillez pas.'

Cette insigne sottise, qui semblait démontrer qu'en effet l'Italie était chose inhumée, à ne ressusciter jamais, fit bondir Michel-Ange. [...]

Le Jour n'est pas fini. Ce rude forgeron, de force colossale, couché sur son marteau, tournant le dos au monde indigne de le voir, devait jeter par-dessus l'épaule un superbe regard. Il était, dans ce deuil, le côté de l'espoir, de l'art, de l'action, de la rénovation future. Mais l'homme était brisé. Michel-Ange laissa ce travail. Et il reste inachevé ${ }^{13}$.

Le monument de la Mort devait être un tombeau [...] Il ne resta de l'œuvre commencée que le Moïse et les Esclaves; ces derniers sont au Louvre (le plâtre du Moise aux Beaux-Arts) [...] Grand souffle et grand esprit ! quel air libre circule ici, hors de toute limite de nations, de temps, de religions! tout l'Ancien Testament y est, mais continu. Et ceci le déborde. Du christianisme, nul signe ${ }^{14}$.

Voilà comment écrivait, comment pensait, en ce temps, en ce lieu, le visiteur inspiré par les plâtres de Michel-Ange. Rhétorique et poétique, l'énergie michélangélienne et byronienne parlait une langue que tous entendaient, excepté Carpeaux et la fille du soudard. Alexandre Dumas s'adressait au buste en plâtre de Brutus - le moulage est détruit - comme à Lorenzaccio ou à Garibaldi : «Michel-Ange a l'âme de Brutus et l'âme de l'Italie est dans Michel-Ange [...] Il naît républicain et meurt républicain, et, pendant soixante ans de son existence, il est obligé de servir les princes et de flatter les papes » ${ }^{15}$.

Dumas récrivit l'affrontement de Raphaël et de Michel-Ange, devant leurs œuvres mises côte à côte, victoire romantique de la pensée libre sur la foi et la confiance : "L'âme de l'Italie est dans celui qui du Raphaël chrétien et idéaliste fera un Raphaël matérialiste et païen ${ }^{16}$.» Un moulage de l'unique sculpture de Raphaël monte la garde à l'entrée de la chapelle (fig. 9) À propos du Jonas de Sainte-Marie-du-Peuple, par Raphaël et Lorenzetti, John Pope-Hennessy eut une formule terrible qui fera méditer les gypsolâtres :

The only statue Raphael himself designed, the Jonah in the Chigi Chapel in Santa Maria del Popolo, where his cartoon - and very beautiful it must have been - was blurred and weakened by the sculptor Lorenzetto. This is one of the few sculptures in the world whose true merits transpire more clearly from a moulding cast than from the original ${ }^{17}$. 


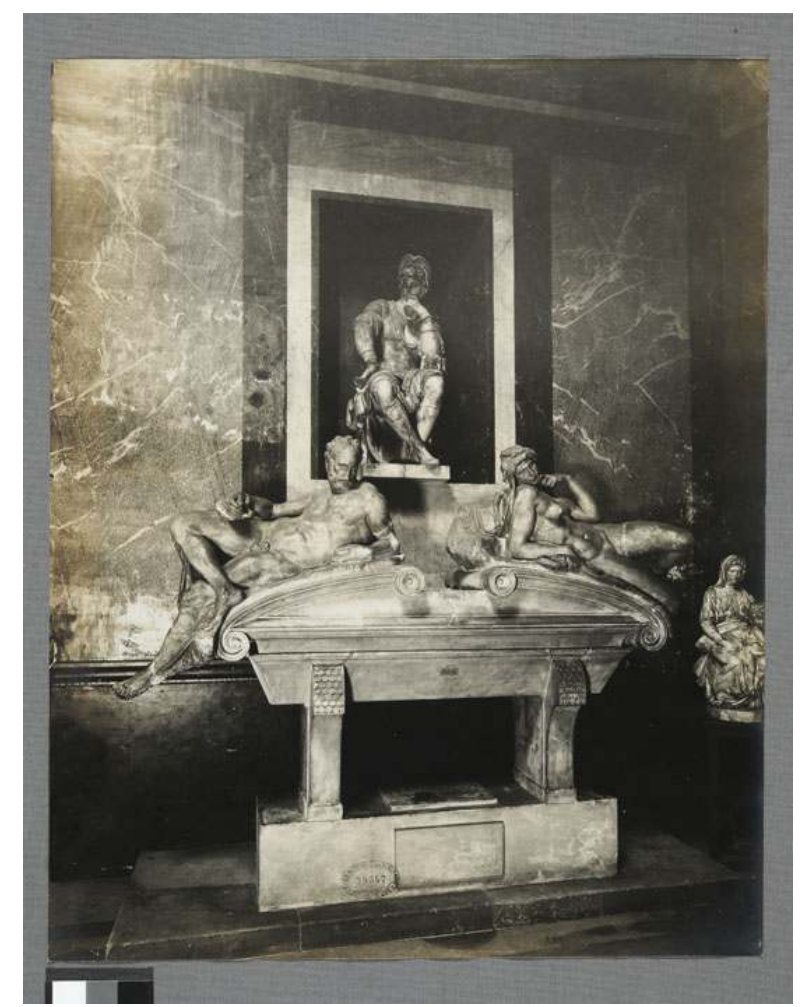

Anonyme. Le Pensieroso, L'Aube et Le Crépuscule, monument de Lorenzo de Medici, moulé par Pappi en 1836, 1913, photographie. Paris, École nationale supérieure des beaux-arts, PH 9191.

Phot. Lapelerie, Jean-Michel. (c) École nationale supérieure des beaux-arts.

17 John Pope-Hennessy vit le Jonas sans passion ni idéologie à l'entrée du lieu où les chefsd'œuvre désespérés, dans la mutation de marbre en plâtre, posent des énigmes à tout sculpteur qui modèle, taille ou cisèle.

On attribua à Michel-Ange tout ce qui contredisait le doux Raphaël : l'Adonis mourant, le $5^{\mathrm{e}}$ esclave, le Garçon accroupi de Saint-Pétersbourg. Pour Alexandre Lenoir, Michel-Ange était sans contredit le plus grand peintre - du Moyen Âge ${ }^{18}$. Ce jugement archaïque naissait de la proximité de la collection de moulages avec la copie, dans la nef, du Jugement dernier; en face du visiteur, l'Enfer régnait, à droite, le Paradis de Ghiberti le rassurait. Un lieu commun faisait de Michel-Ange le disciple fidèle de Dante, et les scènes de Ghiberti, dans l'autre axe de l'église, exprimaient mieux l'humanité ou l'humanisme que les malédictions du Dante. Delacroix comparait dès 1824 le sublime selon Dante et le sublime selon Michel-Ange. Il ne connut guère Michel-Ange que par la collection de l'École, puisqu'il n'alla jamais en Italie; il fit de lui le premier artiste moderne, non le passage du Moyen Âge à la Renaissance, mais le saut du baroque au Romantisme ${ }^{19}$. Où insérer le génie solitaire dans une chronologie? Théophile Gautier jugeait tel morceau indigne de Michel-Ange: "Qu'on nous pardonne l'abominable blasphème que nous proférons : il nous a paru un peu Vanloo $»^{20}$. Ce n'est plus Michel-Ange qui blasphème Notre Seigneur, c'est le rococo qui avilit et souille le Dieu des mécréants. Plus prudent, Eugène Müntz, professeur et conservateur, parlait sans trop d'arrière-pensée du Bacchus du Bargello et du Cupidon du South Kensington, androgyne pourvu de «la délicatesse 
d'une femme ». Il pressentait que la mélancolie, « cette maladie de plus en plus profonde » expliquait ou expliquerait un jour, l'ambiguïté de ces œuvres déroutantes ${ }^{21}$.

\section{Poétique française}

Ils étaient deux poètes, le maitre et le disciple, les deux meilleurs ennemis de l'École de Paris. Théophile Gautier exalta un Michel-Ange en prophète prométhéen, par une série d'articles et de poèmes composés dans sa chapelle : «Le but de l'art ne consiste pas dans l'imitation de la nature. L'art est une création dans la création, et les peintres doivent faire le poème de l'homme et non son histoire $\$^{22}-$ le poème de l'homme défiant le Créateur :

On apporte quatre montagnes de marbre à ce Titan sculptural; il se jette dessus comme un lion sur sa proie [...] demandez-lui ce qu'il fait; il n'en sait rien, il fait un homme, son seul et unique sujet - cela s'appellera, s'il vous plaît, le Crépuscule, la Nuit, le Point du jour, que sais-je ? Car il faut bien un titre et un sujet - pour les sots; mais, en vérité, ce n'est autre chose qu'un homme qui vous montre, celui-ci son dos, celui-là ses bras et ses cuisses sous une attitude impérieuse et magistrale...

Le critique redevenait poète pour conclure par deux alexandrins :

Des chairs comme du marbre et des musculatures

[...] Ils portent ta pensée, ô maître, sur leurs $\operatorname{dos}^{23}$.

21 La transition de Gautier romantique à Baudelaire symboliste, telle qu'elle se réalisa à travers leur inspiration plastique, appartient à l'histoire littéraire. Baudelaire commenta Michel-Ange dans les Fleurs du mal, non dans sa critique d'art. On lui donna la Nuit, il en fit L'Idéal ${ }^{24}$.

«Et toi, grande Nuit, fille de Michel-Ange,

Qui tors paisiblement dans une pose étrange

Tes appas façonnés aux bouches des Titans. »

On lui donna le plâtre, il en fit $L a$ Géante $e^{25}$.

«J'eusse aimé vivre auprès d'une jeune Géante

Comme aux pieds d'une reine un chat voluptueux

$[\ldots]$

Parcourir à loisir ses magnifiques formes ;

Ramper sur le versant de ses genoux énormes,

Dormir nonchalamment à l'ombre de ses seins

Comme un hameau paisible au pied d'une montagne. »

Baudelaire n'alla jamais en Italie. Les historiens de la littérature hésitent entre un Baudelaire inspiré par la gravure et un Baudelaire amateur de moulages. La solution est dans les textes: le critique est un aquafortiste, le poète aspire à l'idéal plastique. Les poèmes michelangéliens que nous citons évoquent les couleurs et les volumes, non les lignes ni les ombres, ce qui nous fait croire que Baudelaire visita l'École des beaux-arts, peut-être avec Théophile Gautier, le poëte impeccable, ou sur ses indications - Jean Prévost pense aussi que Baudelaire connut la sculpture de Michel-Ange à travers les copies de l'École des beaux-arts plutôt que par la gravure ${ }^{26}$.

Auguste Rodin fut trois fois candidat à l'École des beaux-arts, trois fois refusé - honneur aux professeurs qui reconnurent aussi sûrement son génie. Rodin fut au moins admis dans le tempietto de Michel-Ange. Il jeta sur la feuille les dessins allégoriques du Jour et de la Nuit. Puis, pour le frontispice d'une édition posthume des Fleurs du mal, sous les genoux 
de Satan, le Prince de la Nuit, redevenue masculine, il plaça le poète récitant une épave des Fleurs du Mal ${ }^{27}$ :

Vous avez, en secret, baisé ma fesse immonde!

Reconnaissez Satan à son rire vainqueur,

Énorme et laid comme le monde !

Les dessins de Rodin sont peu convaincants, mais son intelligence de Baudelaire et de Michel-Ange donne le vertige. Il est démontré que Rodin a dessiné dans l'École plus longuement qu'à San Lorenzo. Là, avant Rodin, Baudelaire avait vu le Phare Michel-Ange éclairer la voûte maudite. Il avait vu et exalté

[...] des Hercules

Se mêler à des Christs, et se lever tout droits

Des fantômes puissants qui dans les crépuscules

Déchirent leur suaire en étendant leurs doigts ${ }^{28}$.

Et plus tôt encore, cent fois citée au cours du siècle, la Nuit de Michel-Ange avait martelé dans le marbre et dans les esprits l'épigramme, qui déjà prenait les accents de la Beauté baudelairienne : Grato m’è il sonno, e più l'esser di sasso ${ }^{29}$ («Il m'est doux de dormir et plus encore d'être de pierre »).

\section{De plâtre et de marbre}

Gustave Moreau, le lettré professeur de l'École, le maître symboliste, le lecteur de Baudelaire, évoquait encore, dans la chapelle même, le sommeil de Michel-Ange et le "songe de la vie humaine " (dessin de Michel-Ange d'après ses propres compositions): "Toutes ses figures sont fixées dans un geste de somnambulisme idéal [...] le sommeil dans l'attitude ${ }^{30}$.» - le sommeil ou la mort? Quelle erreur : c'était la vieille école qui mourait de ses exercices ressassés, appliquant encore et toujours l'anatomie à écorcher et désosser par le dessin les figures de Michel-Ange, tandis que le Divino se levait enfin de son sommeil de plâtre pour son rêve de pierre. Le Michel-Ange symboliste, à la dimension de l'univers, éclos de la poésie, vola son énergie à la matière. Michelet lisait les Sonnets [Rime] de Buonarroti, il y trouva l'explication de sa puissance, la pensée de Platon: "Chaque soir, il rimait un sonnet, et toujours sur les mêmes textes, sur l'effort impuissant de l'âme pour se sculpter elle-même, se tirer de son bloc, sur la difficulté qu'elle rencontre à dégager du marbre - l'Idée, objet de son désir, son austère fiancée ${ }^{31}$.» Hippolyte Taine, après le Voyage en Italie, enseigna aux élèves de l'École à affronter le marbre :

Rappelez-vous son chef d'œuvre, les quatre statues de marbre placées à Florence sur le tombeau des Médicis [...]. Tout le monde a vu le plâtre de ces statues, mais, à moins d'être venu ici, personne n'a vu leur âme. Je vois là toutes les figures de Dante, Ugolin rongeant le crâne de son ennemi, les damnés qui sortent à demi de leur sépulcre de braise ; mais ceux-ci ne sont point des maudits, ce sont de grandes âmes blessées qui s'indignent justement contre la servitude ${ }^{32}$

- servitude de la chair périssable, chrétienne ou platonicienne. Le sculpteur aussi est un esclave révolté - contre la matière inexorable.

Un court séjour à Florence en 1876 révéla à Rodin le langage du marbre ${ }^{33}$ : «Tout ce que j'ai vu [...] de plâtre, ne donne aucun idée de la sacristie de Saint-Laurent $»^{34}$. Du friable gypse parisien, Rodin avait reçu le symbolisme de Michel-Ange; à Florence, dans le modelé du marbre gradiné, il discerna " l'âme humaine qui voudrait faire éclater la chape de son enveloppe corporelle afin de posséder la liberté sans limite ${ }^{35}$ ». Rodin revint à 
l'École des beaux-arts pour étudier à loisir, sous la Porte du Paradis, les souffrances de Michel-Ange, et pour mieux fermer la Porte de l'Enfer sur le Penseur. Rodin balança entre deux partis pour la Porte de l'Enfer, la présentation du Louvre avec les Esclaves de part et d'autre de la Porta Stanga de Crémone, en marbre, ou le contrepoint, dans l'École, en plâtre, de la Porte du Paradis et de Laurent de Médicis en penseur. Entre le Louvre, San Lorenzo et l'École des beaux-arts, Rodin modula la divine comédie du plâtre et du marbre. À Paris, Taine et Rodin, le maître et l'élève rejeté, avaient habitué leur œil à la matité du plâtre ; ils furent éblouis par l'éclat diaphane du marbre que la pensée du Créateur avait arraché à la falaise de Carrare. Ils quittèrent la caverne michelangélienne ; leur caverne, à tous, poètes et sculpteurs, aura été, pendant quelques heures, quelques mois ou quelques années, cette chapelle. Ombres parmi les ombres plâtreuses, ils y entrevirent, à travers son simulacre, la Beauté, et consacrèrent leur vie et leur œuvre à lui rendre ses clartés éternelles.

Biographie

Longtemps professeur agrégé de grammaire et philologie classiques, Emmanuel Schwartz est conservateur du patrimoine depuis 1995. Il est chargé à l'École des beaux-arts de Paris des peintures, sculptures, originaux, copies et moulages, ainsi que des fragments et maquettes d'architecture. Il étudie en particulier la tradition pédagogique et humaniste héritée de l'Académie, en la replaçant dans le milieu intellectuel parisien. Ses écrits et expositions traitent des liens entre l'art, la littérature et la politique : Les sculptures de l'École des beaux-arts de Paris. Histoire, doctrines, catalogue (Paris 2003). Homère à l'École des beaux-arts (2004-2005, Paris-Princeton-New York). L'École de la liberté, Être artiste à Paris 1648-1817 (Paris, 2009).

\section{BIBLIOGRAPHIE}

BAUDELAIRE, Charles. Les Fleurs du Mal, édition posthume, préfacée par Théophile Gautier. Paris : Lévy frères, 1868.

CHASTEL, André. « Michel-Ange en France ». Dans Fables, Formes, Figures. Paris : Flammarion, 1978, 2 vol.

DELACROIX, Eugène. Journal 1822-1863, éd. par André Joubin. Paris : Plon, 1981.

DEZALLIER D'ARGENVILLE, Antoine-Joseph. Abrégé de la vie des plus fameux peintres. Paris : De Bure, 1762, 4 vol.

DUMAS, Alexandre. «L'École des Beaux-Arts ». Dans Paris-Guide, 1867, I.

ELSEN, Albert and VARNEDOE, J. Kirk. The Drawings of Rodin. Londres : Elek, 1972.

FRÉART DE CHAMBRAY, Roland. Idée de la Perfection de la Peinture démonstrée par les principes de l'art . Paris : École des beaux-arts, 2005, première édition, Le Mans, 1662.

FORGONZI, Flavio, MIMITA LAMBERTI, Maria, RIOPELLE, Christophe. Michelangelo nell'ottocento. Rodin e Michelangelo. Cat. exp. Florence, Casa Buonarroti, 1996. Milan : Charta, 1996.

GAUTIER, Théophile. Fusains et Eaux-fortes. Paris : Charpentier, 1880. 
KORCHANE, Mehdi (dir.) Michel-Ange au siècle de Carpeaux. Cat. exp, Valenciennes, musée des Beaux-Arts, 2012. Milan : Silvana Editoriale, 2012.

LANEYRIE-DAGEN, Nadeije. « Louis Peisse et le 'Musée des Modèles' à l'École des Beaux-Arts ». Bulletin de la Société d'Histoire de l'Art français, année 1985, Paris, 1987, p. 218-241.

LENOIR, Albert. Inventaire général des richesses d'art de la France. Archives du musée des monuments français. Paris : Plon, 1883-1897, 3 vol.

LENOIR, Alexandre. «Le Jugement dernier de Michel-Ange copie exécutée par M. Sigalon ». Journal des artistes, Paris, $11^{\mathrm{e}}$ année, 1837, I, 14 et 28 mai 1837, p. 315-316 et 339-342.

MOREAU, Gustave. L'Assembleur de rêves, écrits complets de Gustave Moreau. Pierre-Louis Mathieu et Jean Paladilhe (éd.). Fontfroide : Fata Morgana, (Bibliothèque artistique et littéraire), 1984.

MÜNTZ, Eugène. Histoire de l'art pendant la Renaissance. Paris : Hachette, 1889-1895. 3 vol. Vol. III. Italie. La Fin de la Renaissance. Michel-Ange. Le Corrège. Les Vénitiens.

PILES, Roger de. Abrégé d'anatomie accommodé aux arts de peinture et de sculpture. Paris : chez J. Mariette, 1733. Planches gravées par François Tortebat, $1^{\mathrm{e}}$ édition 1668.

POPE-HENNESSY, John. Italian High Renaissance \& Baroque Sculpture, An Introduction to Italian Sculpture. Oxford : Phaidon, 1963.

PRÉVOST, Jean. Baudelaire. Paris : Mercure de France, 1953.

STENDHAL. Voyages en France, éd. Victor Del Litto. Paris : Gallimard (bibliothèque de la Pléiade), 1992.

TAINE, Hippolyte. Voyage en Italie. Paris : Julliard, 1965, 2 vol., II ; première édition, Paris, Hachette, 1866.

THACKERAY, William M. On the French School of Painting: with appropriate anecdotes, illustrations, and philosophical disquisitions in a letter to Mr MacGilp, of London (The Paris sketch book of Mr. M. A. Titmarsh). New York : 1852, D. Appleton, 2 vol., I, p. 71, originally published, The Fraser's Magazine, December 1839.

\section{NOTES}

1. - Sur Michel-Ange et la France avant le XIX ${ }^{\mathrm{e}}$ siècle, voir CHASTEL, André. « Michel-Ange en France »; Dans Fables, Formes, Figures. Paris : Flammarion, 1978, 2 vol., II, p. 189-206.

2. - FRÉART DE CHAMBRAY, Roland. Idée de la Perfection de la Peinture démonstrée par les principes de l'art. Paris : École des beaux-arts, 2005, p. 227, première édition, Le Mans, 1662, p. 66-67.

3. - DEZALLIER D'ARGENVILLE, Antoine-Joseph. Abrégé de la vie des plus fameux peintres. Paris : De Bure, 1762, 4 vol., I, p. 135.

4. - PILES, Roger de. Abrégé d'anatomie accommodé aux arts de peinture et de sculpture. Paris : chez J. Mariette, 1733. Planches gravées par François Tortebat, $1^{\mathrm{e}}$ édition 1668, préface, non paginée.

5. - LENOIR, Alexandre. "Projet de catalogue du Dépôt provisoire des Petits-Augustins » 19 thermidor an II (16 août 1794). Dans Inventaire général des richesses d'art de la France, archives du musée des monuments français. Paris : Plon, 1886, 3 vol., vol. 2, p. 180.

6. - LANEYRIE-DAGEN, Nadeije. «Louis Peisse et le 'Musée des Modèles' à l'École des Beaux-Arts ». Bulletin de la Société d'Histoire de l'Art français, année 1985. Paris, 1987, p. 218-241, p. 231.

7. - STENDHAL. Voyages en France, éd. Victor Del Litto. Paris: Gallimard (bibliothèque de la Pléiade), 1992, p. 124.

8. - LANEYRIE-DAGEN, N., op. cit., 1987, p. 231-232. 
9. - DELACROIX, Eugène. Cuvres littéraires. Paris: Grès, 1923, II, p. 217-224, "Sur le Jugement dernier », publié d'abord dans la Revue des Deux-Mondes, le $1^{\mathrm{er}}$ août 1837.

10. - THACKERAY, William M. On the French School of Painting: with appropriate anecdotes, illustrations, and philosophical disquisitions in a letter to Mr MacGilp, of London (The Paris sketch book of Mr. M. A. Titmarsh). New York: 1852, D. Appleton, 2 vol., I, p. 71, originally published, The Fraser's Magazine, December 1839.

11. - KORCHANE, Mehdi (dir.). Michel-Ange au siècle de Carpeaux. Cat. exp, Valenciennes, musée des Beaux-Arts, 2012. Milan : Silvana Editoriale, 2012 p. 143.

12. - GAUTIER, Théophile. Fusains et Eaux-fortes. Paris : Charpentier, 1880, p. 124, publication originale, Charte de 1830, feuilleton du 17 mai (18) et 22 mai.

13. - MICHELET, Jules. Renaissance et Réforme. Paris : Robert Laffont, 1982, p. 341, première édition, Histoire de France (VIII, XVI ${ }^{e}$ siècle), La Réforme. Paris: Chamerot, 1855, chap.XIV, «Le sac de Rome ", p. 300-302.

14. - Ibid., 1982, p. 174 et 177-78, édition originale, Histoire de France (VII, XVI ${ }^{e}$ siècle), Renaissance. Paris : Chamerot, 1855, chap. XIV, « De Michel-Ange comme prophète », p. 230.

15. - DUMAS, Alexandre. «L'École des Beaux-Arts ». Dans Paris-Guide, 1867, I, p. 864 et 860. Dumas ou son nègre paraphrasait en 1867 des propos antérieurs de Michelet.

16. - Ibid., I, p. 860.

17. - POPE-HENNESSY, John. Italian High Renaissance \& Baroque Sculpture. An Introduction to Italian Sculpture. Oxford : Phaidon, 1963, Part III, p. 44.

18. - LENOIR, Alexandre. "Le Jugement dernier de Michel-Ange copie exécutée par M. Sigalon ", Journal des artistes, Paris, $11^{\mathrm{e}}$ année, 1837, I, 14 et 28 mai 1837, p.315-316 et 339-342. [signé F]. Reprise de Le Jugement universel de Michel-Ange, par M. Xavier Sigalon : rapport lu à la quatrième classe de l'Institut historique : (Histoire des Beaux-arts). s.l., s.n., 1833.

19. - DELACROIX, Eugène. Journal 1822-1863. éd. par André Joubin. Paris : Plon, 1981, p. 77 et tout le commentaire « Sur le Jugement dernier », voir note supra.

20. - GAUTIER, Th., op. cit., 1880, p. 142.

21. - MÜNTZ, Eugène. Histoire de l'art pendant la Renaissance. Paris : Hachette, 1895, 3 vol. III. Italie. La Fin de la Renaissance. Michel-Ange. Le Corrège. Les Vénitiens, p. 383-384.

22. - Ibid., p. 138 et 125.

23. - Ibid.

24. - BAUDELAIRE, Charles. «L'Idéal ». Les Fleurs du Mal, XIX.

25. - Ibid., « La Géante ». Les Fleurs du Mal, XVIII.

26. - PRÉVOST, Jean. Baudelaire. Paris : Mercure de France, 1953, rééd. 1964, p. 112 et 147. Le livre de Prévost est entré dans la mythologie romanesque de la France en raison de la qualité de ses analyses, et aussi parce que son auteur, résistant et maquisard, fut abattu par les Allemands en 1944, avant qu'il ne pût achever et publier son ouvrage.

27. - Id., « L'Imprévu ». Les Fleurs du Mal, Épaves, III.

28. - Id., « Les Phares ». Les Fleurs du Mal, VI.

29. - DELACROIX, E., op. cit., 1923, II, 44, réédition des « Essais sur les artistes célèbres ». Revue de Paris, 1830.

30. - MOREAU, Gustave. L'Assembleur de rêves, écrits complets de Gustave Moreau. Pierre-Louis Mathieu et Jean Paladilhe (éd.). Fontfroide : Fata Morgana (Bibliothèque artistique et littéraire), 1984 , p. 220 , note 9 , et p. 222,230 et 306 . Le dessin allégorique Le Songe de la vie humaine appartient au Courtauld Institute de Londres.

31. - MICHELET, J., op. cit., 1982, p. 174.

32. - TAINE, Hippolyte. Voyage en Italie. Paris : Julliard, 1965, 2 vol., II, p. 150-151, première édition, Paris, Hachette, 1866. 
33. - FORGONZI, Flavio, MIMITA LAMBERTI, Maria, RIOPELLE, Christophe. Michelangelo nell'Ottocento. Rodin e Michelangelo. Cat. exp. Florence, Casa Buonarroti, 1996. Milan : Charta, 1996, p. 32. Voir ELSEN, Albert and VARNEDOE, J. Kirk. The Drawings of Rodin. London : Elek, 1972, p. 43.

34. - RODIN, Auguste. Correspondance, 1985-1992. Paris : Musée Rodin, I, p. 33-34.

35. - Ibid., II, p. 216.

\section{RÉSUMÉS}

Thiers créa dans l'École des beaux-arts un sanctuaire de moulages dédié à Michel-Ange. Cette collection révéla les affinités entre la terribilità de Michel-Ange et le romantisme politique et irréligieux de Stendhal et Michelet. Elle eut des échos dans la poésie de Théophile Gautier et de Baudelaire. Carpeaux et Rodin, sensibles à la puissance de Michel-Ange, conçurent là d'audacieuses inventions. Taine se servit du plâtre parisien pour enseigner à ses élèves le symbolisme tragique des originaux en marbre.

In the 1840s, the minister Adolphe Thiers created a 'shrine' at the École des beaux-arts in Paris, made up of a collection of casts after Michelangelo. This collection showed the affinities between Michelangelo's terribilità and the political and irreligious romanticism of Stendhal and Michelet. It had echoes in the poetry of Théophile Gautier and Baudelaire. For the sculptors Carpeaux and Rodin, Michelangelo's works inspired audacious inventions. Taine used these Parisian plaster casts to show to his pupils the tragic symbolism of the original marbles.

INDEX

Mots-clés : Michel-Ange, plâtre, moulage, Musée des monuments français (des PetitsAugustins), Muséum du Louvre, église et couvent

Keywords : Michelangelo, plaster casts, church and convent

\section{AUTEUR}

\section{EMMANUEL SCHWARTZ}

Conservateur des moulages, École des beaux-arts de Paris emmanuel.schwartz@ensba.fr 